\title{
EFFECTIVENESS INTO PRACTICE
}

\section{Nicholas Phin \\ Project Director \\ Health Evidence Bulletins \\ University of Wales College of Medicine, Cardiff}

The almost exponential increase in the amount of information from research that is available to health professionals relating to new treatments, therapies and our understanding of disease processes is creating enormous problems for practitioners and policy makers alike. A new initiative being developed in Wales seeks to provide these two groups with straightforward statements about health which have clearly referenced evidence that has been critically appraised and subjected to a formal literature search across a wide range of sources. Oral health was identified as a key area for inclusion in the project and an oral health bulletin has been produced under the direction of Dr A.L. Glenn, a consultant in dental public health.

In 1995, it was estimated that a general physician needed to examine 19 articles a day, 365 days a year, in order to extract the information required to keep up-to-date in his or her speciality. There is no reason to believe this situation is any different in 1999 and, in fact, with the much greater emphasis on electronic communication, it may be worse. This type of information overload has implications not only for an individual's professional requirement to maintain acceptable standards of care and expertise, but also for the bodies responsible for setting and monitoring the professional standards and competencies of practitioners. In addition, it creates real problems for those trying to develop and implement policy: does the new treatment $\mathrm{X}$ offer any advantages over current practice; should a change in practice be supported, and when is it likely to be superseded?

Therefore, it was felt to be essential that clinicians and policy makers have easy access to clear and succinct statements about health issues that were backed up by references that were up-to-date, indicated the strength of the evidence, had been rigorously and critically appraised and had been subjected to a formal literature search. The Health Evidence Bulletins, born from the work of the original Welsh Health Planning Forum, seeks to provide such a source of information, both in paper format and in electronic format through the World Wide Web. The layout of the bulletins was designed for easy reading and searching, and the hypertext links built into the electronic version enable many of the references cited to be accessed directly on screen.

Eight subject areas were chosen for the Health Evidence Bulletin relating to oral health:

- tooth decay

- periodontal diseases

- dentofacial anomalies

- oral cancer

- TM joint disorders and complex facial pain

- tooth wear and hypersensitivity

- dental injuries

- inherited dental anomalies.

The papers produced by a systematic literature search were first appraised by a clinical team, and then their products were scrutinized by external reviewers in an effort to both minimise bias and ensure that the final product was as robust as possible. The production of this current bulletin is seen only as a first stage. It is intended that the process will be repeated, incorporating new evidence as it becomes available and expanding the range of topics covered.

The Oral Health bulletin can be accessed through the Internet at www.hebw.uwcm.ac.uk, where paper copies may also be ordered. Nicholas Phin can be contacted by email atweightmana@cardiff.ac.uk. w

\section{ORAL HEALTH:WELSH HEALTH EVIDENCE BULLETIN}

\section{Clive Wright}

Director of Health Promotion, Research

and Specialist Services,

Dental Health Services Victoria

Oral Health is one in a series of 12 publications initiated by the Protocol Enhancement Project, Wales Office of Research and Development for Health and Social Care. The aim of the Health Evidence Bulletins-Wales is to provide 'the best current evidence across a broad range of evidence types and subject areas'.
The Protocol Enhancement Project for the Oral Health health evidence bulletin was developed and edited by both an internal oral health review group (namely, academic and public health expertise drawn from Wales) and an external advisory group drawn from prestigious dental academic institutions of the United Kingdom.

The randomised controlled trial (RCT) has been the 'gold standard' for evidence-based health research, and the bulletins include RCTs where available, but also sift through 'high quality evidence' from observational and 
other studies. In this sifting process they use both an adaptation of the Bandolier hierarchy of evidence type, ${ }^{1}$ and the health benefit notation system described by Enkin et al (1995). ${ }^{2}$

The protocol provides clear, contemporary statements on an oral health intervention, together with an indication of the strength of evidence to support the statement (hierarchy plus potential benefit), and citation of the source of the evidence. For example, the statement 'Fluoride varnishes reduce dental caries and are particularly recommended for special needs groups' cites evidence from two sources Helfeustein and Steiner ${ }^{3}$ [Type I evidence-meta-analysis] and Oral Health ${ }^{4}$ [Type V evidence-expert opinion] and describes the health gain as 'beneficial-evidence clearly demonstrated'.

The health evidence bulletins make an important departure from the 'gold standard' RCT evidence base. They argue that many health issues do not lend themselves to investigation by RCT and that, by valuing evidence from RCTs more highly, interventions with limited effectiveness might be judged more worthy than those based on observation. Further, that information 'assigned as Type $\mathrm{V}$ evidence includes important reports of recommendations which should rightly be highly regarded' (author's emphasis). Many epidemiologists and quantitative purists may find this approach counter to the principle of the Cochrane Collaboration. Yet, in health fields such as dentistry and oral health, the contribution that observational and qualitative research has made to oral health gain is immeasurable. It is impossibile to conduct a randomised clinical trial of water fluoridation. The closest one can get is a well controlled and rigorously conducted observational trial. Further, RCTs are extremely expensive. Oral health research, especially oral health promotional research, does not compete well for the funding for highly extensive and expensive studies. Consequently, opportunity for applying RCT principles to measuring, for example, the impact of frequency and form of sugar consumption on dental caries, or the impact of educating nursing home carers in the provision of oral health care to the elderly may be both impractical and inappropriate. More cost-appropriate evaluation may well lie in soundly structured qualitative, or less rigidly constrained quantitative, methods. Therefore, the Oral Health bulletin makes a valuable contribution to evidenced-based dentistry in the public health context. However, readers should be aware that the goal posts have been moved.

Oral Health reports more than 100 interventional statements in eight dental subject areas: tooth decay; periodontal diseases; dentofacial anomalies; oral cancer; temporomandibular joint disorders and complex facial pain; tooth wear and hypersensitivity; dental injuries; and inherited dental anomalies. The statements cover areas in which there is professional controversy and important dental public health implications, for example, in the value of periodic dental examinations and the use of dental amalgam.

The Protocol Group supported the statement 'Regular clinical examination is recommended for the early detection of tooth decay, with radiographs (x-rays) to detect caries not visible on examination. The interval between successive clinical and radiographic examinations should vary according to the caries susceptibility of the individual', with a health benefit notation indicating that the intervention was beneficial and the effectiveness clearly demonstrated. Two citations, one providing Type IV evidence (well designed observational studies) and the other Type $\mathrm{V}$ evidence (expert opinion; influential reports and studies), supported the notation. Likewise, the statement 'Dental amalgam is an effective filling material. Amalgam restorations do not appear to be hazardous to the general health of the population' was supported by the Protocol group with a health benefit notation indicating a beneficial heath outcome. Three citations were used to support the statement at three levels of evidence: Type III- 'well designed interventional studies without randomisation'Type IV and Type V.

Neither of these two important dental public health subject areas are measured easily by RCTs. Yet, the best research evidence available must be used by planners and decisionmakers to ensure that rationality continues in the provision of public dental health services.

Statements within Oral Health are appraised clearly and critically. They represent the best evidenced-based practice available in dentistry measured against a defensible standard. This health evidence bulletin is strongly recommended to all dental practitioners and dental public health workers.

\section{REFERENCES}

1. Bandolier system. See: www.jr2.ox.ac.uk/Bandolier/band6/ b6-5.html.

2. Enkin M, Keirse MJNC, Renfrew M, Neilson J. A guide to effective care in pregnancy and childbirth, 2nd edn. Oxford: Oxford University Press, 1995: 389-390.

3. Helfeustein U, Steiner M. Fluoride varnishes (Duraphat): a meta-analysis. Community Dent Oral Epidemiol 1994; 22: $1-5$.

4. Welsh Health Planning Forum. Oral health: Protocol for investment in health gain. Cardiff: Welsh Office NHS Directorate, November 1992. it 